\title{
Numerical error for SDE: Asymptotic expansion and hyperdistributions
}

\author{
Paul Malliavin ${ }^{\mathrm{a}}$, Anton Thalmaier ${ }^{\mathrm{b}}$ \\ a 10, rue Saint Louis en l'Isle, 75004 Paris, France \\ ${ }^{\mathrm{b}}$ Université d'Évry, laboratoire d'analyse et probabilité, bd François Mitterrand, 91025 Evry cedex, France \\ Received 13 March 2003; accepted 1 April 2003 \\ Presented by Paul Malliavin
}

\begin{abstract}
The principal part of the error in the Euler scheme for an SDE with smooth coefficients can be expressed as a generalized Watanabe distribution on Wiener space. To cite this article: P. Malliavin, A. Thalmaier, C. R. Acad. Sci. Paris, Ser. I 336 (2003).

(C) 2003 Académie des sciences. Published by Éditions scientifiques et médicales Elsevier SAS. All rights reserved.

\section{Résumé}

Erreur du schéma d'Euler pour une EDS et hyperdistribution. La partie principale de l'erreur dans l'intégration par le schéma d'Euler d'une EDS avec des coefficients réguliers est une distribution de Watanabe généralisée. Pour citer cet article : P. Malliavin, A. Thalmaier, C. R. Acad. Sci. Paris, Ser. I 336 (2003).

(C) 2003 Académie des sciences. Published by Éditions scientifiques et médicales Elsevier SAS. All rights reserved.
\end{abstract}

\section{Algebraic computation of the error}

Asymptotic expansion depends upon the choice of a topology for measuring the size of the remainder term; either a norm topology, or on the opposite side, some weak topology may be chosen. The "weakest" among weak topologies are topologies on spaces of distributions: this will be the point of view of this Note. Our main concern is to establish asymptotic expansions "upstairs", that is on the probability space itself. We shall deal with the $\mathbb{R}^{d}$-valued SDE

$$
\mathrm{d} \xi_{W}(t)=\sum_{k=1}^{n} A_{k}\left(\xi_{W}(t)\right) \mathrm{d} W^{k}+A_{0}\left(\xi_{W}(t)\right) \mathrm{d} t ; \quad \xi_{W}\left(t^{0}\right)=\xi_{0}
$$

where $W$ denotes $n$-dimensional Brownian motion on Wiener space $\mathcal{W}^{n}$ and $A_{0}, A_{1}, \ldots, A_{n}$ are smooth bounded vector fields on $\mathbb{R}^{d}$ with bounded derivatives of all orders.

E-mail addresses: sli@ccr.jussieu.fr (P. Malliavin), anton.thalmaier@maths.univ-evry.fr (A. Thalmaier). 
Given $\varepsilon>0$, the Euler scheme of mesh $\varepsilon$ is defined by the following recursion formula:

$$
\begin{aligned}
\xi_{W_{\varepsilon}}(q \varepsilon)-\xi_{W_{\varepsilon}}((q-1) \varepsilon)= & \sum_{k=1}^{n} A_{k}\left(\xi_{W_{\varepsilon}}((q-1) \varepsilon)\right)\left[W^{k}(q \varepsilon)-W^{k}((q-1) \varepsilon)\right] \\
& +A_{0}\left(\xi_{W_{\varepsilon}}((q-1) \varepsilon)\right) \varepsilon, \quad \xi_{W_{\varepsilon}}(t)=\xi_{0} \quad \text { for } t \in\left[t_{\varepsilon}^{0}, t^{0}\right],
\end{aligned}
$$

where for $t \geqslant 0$ we use the notation $t_{\varepsilon}=[t / \varepsilon] \varepsilon$ with $[a]$ the largest integer $\leqslant a$. The Euler scheme for all times is then defined as solution of the delayed SDE

$$
\mathrm{d} \xi_{W_{\varepsilon}}(t)=\sum_{k=1}^{n} A_{k}\left(\xi_{W_{\varepsilon}}\left(t_{\varepsilon}\right)\right) \mathrm{d} W^{k}(t)+A_{0}\left(\xi_{W_{\varepsilon}}\left(t_{\varepsilon}\right)\right) \mathrm{d} t \quad \text { for } t \geqslant t^{0}, \quad \xi_{W_{\varepsilon}} \mid\left[t_{\varepsilon}^{0}, t^{0}\right]=\xi_{0} .
$$

The remainder term $\theta_{\varepsilon}(t):=\xi_{W_{\varepsilon}}(t)-\xi_{W}(t)$ satisfies the SDE

$$
\mathrm{d} \theta_{\varepsilon}=\sum_{k=1}^{n}\left[A_{k}\left(\xi_{W}(t)+\theta_{\varepsilon}(t)\right)-A_{k}\left(\xi_{W}(t)\right)\right] \mathrm{d} W^{k}(t)+\left[A_{0}\left(\xi_{W}(t)+\theta_{\varepsilon}(t)\right)-A_{0}\left(\xi_{W}(t)\right)\right] \mathrm{d} t+\mathrm{d} \chi(t),
$$

where $\theta_{\varepsilon}\left(t^{0}\right)=0$ and

$$
\mathrm{d} \chi(t):=\sum_{k=1}^{n}{ }^{\varepsilon} \mathcal{R}_{k}(t) \mathrm{d} W^{k}(t)+{ }^{\varepsilon} \mathcal{R}_{0}(t) \mathrm{d} t, \quad{ }^{\varepsilon} \mathcal{R}_{k}(t):=A_{k}\left(\xi_{W_{\varepsilon}}\left(t_{\varepsilon}\right)\right)-A_{k}\left(\xi_{W_{\varepsilon}}(t)\right) .
$$

Let $\mathbf{A}_{k}$ be the $d \times d$ matrix defined by differentiating the components of the vector field $A_{k}$ with respect to the coordinate vector fields; then almost surely the derivative of the solution $\xi_{W_{\varepsilon}}(t)$ of (2) with respect to the initial data $\xi_{0}$ defines a random flow of diffeomorphisms; its Jacobian is given by the matrix-valued delayed SDE

$$
\mathrm{d} J_{t \leftarrow t^{0}}^{W_{\varepsilon}}=J_{t \leftarrow t^{0}}^{W_{\varepsilon}}\left(\sum_{k=1}^{n} \mathbf{A}_{k}\left(\xi_{W}\left(t_{\varepsilon}\right)\right) \mathrm{d} W^{k}+\mathbf{A}_{0}\left(\xi_{W}\left(t_{\varepsilon}\right)\right) \mathrm{d} t\right), \quad t \geqslant t^{0},
$$

where $J_{t \leftarrow t^{0}}^{W_{\varepsilon}}=$ id for $t \in\left[t_{\varepsilon}^{0}, t^{0}\right]$. The derivatives of ${ }^{\varepsilon} \mathcal{R}_{k}(t)$ may be computed in terms of the Jacobian matrix:

$$
D_{\tau, \ell}{ }^{\varepsilon} \mathcal{R}_{k}(t)=1_{\{\tau \leqslant t\}}\left(\mathbf{A}_{k}\left(\xi_{W_{\varepsilon}}\left(t_{\varepsilon}\right)\right) J_{t \leftarrow \tau}^{W_{\varepsilon}}\left(A_{\ell}\right)-\mathbf{A}_{k}\left(\xi_{W_{\varepsilon}}(t)\right) J_{t \leftarrow \tau}^{W_{\varepsilon}}\left(A_{\ell}\right)\right)
$$

the derivatives $D_{\tau, \ell} \theta_{\varepsilon}(t)=: u(t)$ are computed by differentiating (3). We get

$$
\mathrm{d} u-\sum_{k=1}^{n} \mathbf{A}_{k}\left(\xi_{W}+\theta_{\varepsilon}\right) u \mathrm{~d} W^{k}-\mathbf{A}_{0}\left(\xi_{W}+\theta_{\varepsilon}\right) u \mathrm{~d} t=: \mathrm{d} \Gamma \equiv \sum_{k=1}^{n} \Gamma_{k} \mathrm{~d} W^{k}+\Gamma_{0} \mathrm{~d} t
$$

where $\Gamma_{0}, \Gamma_{1}, \ldots, \Gamma_{n}$ may be computed using (4) and standard computations of derivatives along the stochastic flow to SDE (1). Then, by Itô's formula, the following version of the Lagrange formula (variation of constants) is established: in terms of the compensation vector field $Z:=\sum_{k=1}^{n} \mathbf{A}_{k} \Gamma_{k}$, we have

$$
u(t)=J_{t \leftarrow t^{0}}\left[\int_{t^{0}}^{t} J_{t^{0} \leftarrow \tau}(\mathrm{d} \Gamma(\tau)-Z(\tau) \mathrm{d} \tau)\right],
$$

where the Itô stochastic integral inside the brackets has to be computed first. We introduce a parameter $\lambda \in[0,1]$ and define ${ }^{\lambda} \theta$ as the solution of the SDE

$$
\begin{aligned}
\mathrm{d}^{\lambda} \theta(t)= & \sum_{k=1}^{n}\left[A_{k}\left(\xi_{W}(t)+{ }^{\lambda} \theta(t)\right)-A_{k}\left(\xi_{W}(t)\right)\right] \mathrm{d} W^{k}(t) \\
& +\left[A_{0}\left(\xi_{W}(t)+{ }^{\lambda} \theta(t)\right)-A_{0}\left(\xi_{W}(t)\right)\right] \mathrm{d} t+\lambda \mathrm{d} \chi(t), \quad{ }^{\lambda} \theta\left(t^{0}\right)=0 ;
\end{aligned}
$$


as ${ }^{0} \theta(t)=0$ for all $t$, denoting $\frac{\mathrm{d}}{\mathrm{d} \lambda}{ }^{\lambda} \theta={ }^{\lambda} u$, we have $\theta_{\varepsilon}=\int_{0}^{1} \lambda u \mathrm{~d} \lambda$. By differentiating (7) with respect to $\lambda$, we get the following linear SDE for ${ }^{\lambda} u$ :

$$
\mathrm{d}^{\lambda} u=\mathrm{d}^{\varepsilon} \mathcal{Q} \cdot{ }^{\lambda} u+\mathrm{d} \chi,
$$

where $\mathrm{d}^{\varepsilon} \mathcal{Q}=\sum_{k=1}^{n} \mathbf{A}_{k}\left(\xi_{W}+{ }^{\lambda} \theta\right) \mathrm{d} W^{k}+\mathbf{A}_{0}\left(\xi_{W}+{ }^{\lambda} \theta\right) \mathrm{d} t$.

\section{Diagonal-regular functions and diagonal-distributions on Wiener space}

We consider the diagonal of $\mathbb{R}^{r}$ which is the 1-dimensional subspace $\gamma=\left\{\tau_{1}=\cdots=\tau_{r}\right\}$; let $\Phi^{r}$ be the quotient map $\mathbb{R}^{r} \mapsto \mathbb{R}^{r} / \gamma$. A function $U \in L^{2}\left(\mathbb{R}^{r}\right)$ is said to be diagonal-continuous if $\mathbb{E}^{\Phi^{r}}\left[U^{2}\right]$ is a continuous function, where $\mathbb{E}^{\Phi^{r}}$ denotes the conditional expectation under $\Phi^{r}$; the space ${ }^{\gamma} D_{r}^{p}$ is the subspace of $D_{r}^{p}$ such that derivatives up to the order $r$ are diagonal-continuous. We define a diagonal Sobolev norm

$$
\|f\|_{\gamma_{D_{r}}^{p}}^{p}=\mathbb{E}\left[|f|^{p}+\sum_{j=1}^{r}\left\|E^{\Phi^{j}}\left|D_{\tau_{1}, \ldots, \tau_{j}} f\right|^{2}\right\|_{L^{\infty}\left(\mathbb{R}^{j} / \gamma\right)}^{p / 2}\right] .
$$

We remark that solutions of SDE with infinitely bounded differentiable coefficients give rise to functionals belonging to ${ }^{\gamma} D_{r}^{p}$. A diagonal-distribution (see [8]) is a linear form on ${ }^{\gamma} D_{r}^{p}$ such that $|\langle f, S\rangle| \leqslant c\|f\|^{\gamma} D_{r}^{p}$.

\section{Estimation of the error in Sobolev norm of positive order}

We get the following estimates of the remainder term in the $L^{p}$-norm, resp. ${ }^{\gamma} D_{r}^{p}$-Sobolev norm; see $[2,6,7]$ for related results.

Proposition 3.1. For any $p \in] 1, \infty[$ and any integer $r>0$, we have

$$
\left(\mathbb{E}\left[\sup _{t \in\left[t^{0}, T\right]}\left|\theta_{\varepsilon}(t)\right|_{\mathbb{R}^{d}}^{p}\right]\right)^{1 / p}=\mathrm{O}(\sqrt{\varepsilon}) ; \quad \sup _{t \in\left[t^{0}, T\right]}\left\|\theta_{\varepsilon}(t)\right\|_{\gamma^{\prime}}=\mathrm{O}(\sqrt{\varepsilon}) .
$$

Proof. Denote by ${ }^{\varepsilon} J_{t \leftarrow \tau}^{W}$ the solution to the linear equation (8) with initial condition ${ }^{\varepsilon} J_{\tau \leftarrow \tau}^{W}=\mathrm{id}$; as its coefficients $\mathbf{A}_{k}$ are bounded, we have uniformly with respect to $\varepsilon$ :

$$
\mathbb{E}\left[\sup _{t, \tau \in\left[t^{0}, T\right]}\left|{ }^{\varepsilon} J_{t \leftarrow \tau}^{W}\right|^{2 p}\right] \leqslant c_{p}<\infty
$$

by the same reason the compensation vector field $Z$ is bounded in $L^{p}$; we get $\mathbb{E}\left[\left.\left.\right|^{\varepsilon} \mathcal{R}_{k}\right|_{\mathbb{R}^{d}} ^{2 p}\right]=\mathrm{O}\left(\varepsilon^{p}\right)$. Using (6), we have

$$
\lambda u(t)={ }^{\varepsilon} J_{t \leftarrow t^{0}}^{W}\left[\int_{t^{0}}^{t} \sum_{k=1}^{n}{ }^{\varepsilon} J_{t^{0} \leftarrow \tau}^{W}\left({ }^{\varepsilon} \mathcal{R}_{k}(\tau)\right) \mathrm{d} W^{k}(\tau)+{ }^{\varepsilon} J_{t^{0} \leftarrow \tau}^{W}\left({ }^{\varepsilon} \mathcal{R}_{0}(\tau)-Z(\tau)\right) \mathrm{d} \tau\right] .
$$

Consequently $D_{\tau, k} \theta$ satisfies an SDE of the same nature as (3); the second part of (9) is verified along the same lines.

Corollary 3.1 (cf. [4]). The Euler scheme converges quasi-surely. 


\section{Asymptotic expansion in terms of a diagonal-distribution}

We localize the method of Section 3 by using a Taylor expansion at $\lambda=0$; denoting the second derivatives by ${ }^{\lambda} v:=(\mathrm{d} / \mathrm{d} \lambda)^{\lambda} u$ we have by means of $(9)$

$$
\theta={ }^{\circ} u+\frac{1}{2}^{\circ} v+\mathrm{o}(\varepsilon) .
$$

The question of asymptotic expansion of $\theta$ as $o(\varepsilon)$ is therefore reduced to the asymptotic expansion of ${ }^{\circ} u,{ }^{\circ} v$ which we abbreviate as $u, v$. We compute $u$ from ( 8 ) for $\lambda=0$ which realizes a computation along the path of the original diffusion. In the same way differentiating equation (8) with respect to $\lambda$ and letting $\lambda=0$, the terms

$$
\mathcal{R}_{k}^{1}(t):=\sum_{\ell, p=1}^{d} \frac{\partial^{2} A_{k}}{\partial \xi^{\ell} \partial \xi^{p}}\left(\xi_{W}(t)\right) u^{\ell}(t) u^{p}(t), \quad Z^{1}(t):=\sum_{k=1}^{n}\left(\mathbf{A}_{k} \cdot \mathcal{R}_{k}^{1}\right)\left(\xi_{W}(t)\right)
$$

appear, and we get

$$
v(t)=J_{t \leftarrow t^{0}}^{W}\left[\int_{t^{0}}^{t} \mathrm{~d} \tau \sum_{k=1}^{n} J_{t^{0} \leftarrow \tau}^{W}\left(\mathcal{R}_{k}^{1}(\tau)\right) \mathrm{d} W^{k}(\tau)+J_{t^{0} \leftarrow \tau}^{W}\left(\mathcal{R}_{0}^{1}(\tau)-Z^{1}(\tau)\right)\right] .
$$

Theorem 4.1 (Rate of weak convergence). There exist $\mathbb{R}^{d}$-valued functions $a_{k \ell}, b_{k}, c$ on $\mathbb{R}^{d}$, computable in terms of the coefficients of (1) and its first fourth derivatives, such that for any $f \in{ }^{\gamma} D_{3}^{\infty-0}$,

$$
\lim _{\varepsilon \rightarrow 0} \frac{1}{\varepsilon} \mathbb{E}\left[\theta_{\varepsilon}(t) f\right]=\int_{t^{0}}^{t} \mathbb{E}\left[\sum_{k, \ell}^{n} a_{k \ell}\left(\xi_{W}(\tau)\right) D_{\tau, k ; \tau, \ell}^{2} f+\sum_{k=1}^{n} b_{k}\left(\xi_{W}(\tau)\right) D_{\tau, k} f+c\left(\xi_{W}(\tau)\right) f\right] \mathrm{d} \tau .
$$

Remark 1. This result should be compared with $[5,1,3]$.

Proof. Assuming that diagonal distributions $\widetilde{S}^{1}, \ldots, \widetilde{S}^{d}$ of third order are given, we consider the formal expression $S^{q}=\sum_{k}\left(J_{t \leftarrow t^{0}}^{W}\right)_{k}^{q} \widetilde{S}^{k}$. As the coefficients of the matrix $J_{t^{0} \leftarrow t}^{W}$ belong to ${ }^{\gamma} D_{3}^{\infty-0}$ and as the space of third order diagonal distributions is a module over the algebra ${ }^{\gamma} D_{3}^{\infty-0}$, we deduce that $S$ is a diagonal distribution of third order. Defining $\tilde{u}(t):=J_{t^{0} \leftarrow t}^{W} u(t)$ and $\tilde{v}(t):=J_{t^{0} \leftarrow t}^{W} v(t)$, we are reduced to find a diagonal distribution $\widetilde{S}$ such that

$$
\lim _{\varepsilon \rightarrow 0} \frac{1}{\varepsilon} \mathbb{E}[\tilde{u}(t) f]=\langle f, \widetilde{S}\rangle, \quad \lim _{\varepsilon \rightarrow 0} \frac{1}{\varepsilon} \mathbb{E}[\tilde{v}(t) f]=0 .
$$

To short-hand the notation, we write ${ }^{W}(\cdot):=J_{t^{0} \leftarrow \tau}^{W}(\cdot)$; then $\tilde{u}(t)=\tilde{u}_{1}(t)+\tilde{u}_{2}(t)$ where

$$
\tilde{u}_{1}(t):=\int_{t^{0}}^{t} \sum_{k=1}^{n}{ }^{W}\left({ }^{\varepsilon} \mathcal{R}_{k}(\tau)\right) \mathrm{d} W^{k}(\tau), \quad \tilde{u}_{2}(t):=\int_{t^{0}}^{t}{ }^{W}\left({ }^{\varepsilon} \mathcal{R}_{0}(\tau)-Z(\tau)\right) \mathrm{d} \tau .
$$

Using the fact that an Itô integral is a divergence we get

$$
\mathbb{E}\left[f \tilde{u}_{1}(t)\right]=\int_{t^{0}}^{t}\left[\sum_{k=1}^{n}\left(D_{\tau, k} f\right)^{W}\left({ }^{\varepsilon} \mathcal{R}_{k}(\tau)\right)\right] \mathrm{d} \tau ;
$$


by Itô formula applied to the delayed SDE (2) we have

$$
{ }^{\varepsilon} \mathcal{R}_{k}(\tau)=-\int_{\tau_{\varepsilon}}^{\tau} \sum_{s} \mathbf{A}_{k}\left(\xi_{W_{\varepsilon}}(\lambda)\right) \cdot A_{s}\left(\xi_{W_{\varepsilon}}\left(\tau_{\varepsilon}\right)\right) \mathrm{d} W^{s}(\lambda)-\int_{\tau_{\varepsilon}}^{\tau}\left({ }^{\varepsilon} \mathcal{L} A_{k}\right)\left(\xi_{W_{\varepsilon}}(\lambda)\right) \mathrm{d} \lambda,
$$

where

$$
\left({ }^{\varepsilon} \mathcal{L} A_{k}\right)\left(\xi_{W_{\varepsilon}}(\lambda)\right):=\frac{1}{2} \sum_{s, \ell, p} A_{s}^{\ell}\left(\xi_{W_{\varepsilon}}\left(\tau_{\varepsilon}\right)\right) A_{s}^{p}\left(\xi_{W_{\varepsilon}}\left(\tau_{\varepsilon}\right)\right) \frac{\partial^{2} A_{k}}{\partial \xi_{\ell} \partial \xi_{p}}\left(\xi_{W_{\varepsilon}}(\lambda)\right)+\sum_{\ell} A_{0}^{\ell}\left(\xi_{W_{\varepsilon}}\left(\tau_{\varepsilon}\right)\right) \frac{\partial A_{k}}{\partial \xi_{\ell}}\left(\xi_{W_{\varepsilon}}(\lambda)\right) .
$$

We want to eliminate the first term in (13). To this end we remark

$$
\begin{aligned}
& \mathbb{E}\left[\left(D_{\tau, r} f\right)^{W}\left({ }^{\varepsilon} \mathcal{R}_{r}(\tau)\right)\right]=\mathbb{E}\left[\mathbb{E}^{\mathcal{N}_{\tau}}\left(D_{\tau, r} f\right)^{W}\left({ }^{\varepsilon} \mathcal{R}_{r}(\tau)\right)\right] ; \\
& \mathbb{E}\left[\mathbb{E}^{\mathcal{N}_{\tau_{\varepsilon}}}\left(D_{\tau, r} f\right) \int_{\tau_{\varepsilon}}^{\tau} \sum_{s} \mathbf{A}_{k}\left(\xi_{W_{\varepsilon}}(\lambda)\right) \cdot A_{s}\left(\xi_{W_{\varepsilon}}\left(\tau_{\varepsilon}\right)\right) \mathrm{d} W^{s}(\lambda)\right]=0 .
\end{aligned}
$$

By the Ocone-Karatzas formula, we get

$$
\mathbb{E}^{\mathcal{N}_{\tau}}\left[D_{\tau, r} f\right]-\mathbb{E}^{\mathcal{N}_{\tau_{\varepsilon}}}\left[D_{\tau, r} f\right]=\sum_{k=1}^{n} \int_{\tau_{\varepsilon}}^{\tau} \mathbb{E}^{\mathcal{N}_{\lambda}}\left(D_{\tau, r ; \lambda, k}^{2} f\right) \mathrm{d} W^{k}(\lambda)
$$

and with ${ }^{W}(\cdot)$ analogously as above,

$$
\begin{aligned}
\mathbb{E} & {\left[f \tilde{u}_{1}(t)\right] } \\
& =\mathbb{E}\left[\int_{t^{0}}^{t} \mathrm{~d} \tau \int_{\tau_{\varepsilon}}^{\tau} \mathrm{d} \lambda\left(\sum_{r, k=1}^{n}\left(D_{\tau, r ; \lambda, k}^{2} f\right)^{W}\left(\mathbf{A}_{r}\left(\xi_{W_{\varepsilon}}(\lambda)\right) \cdot A_{k}\left(\xi_{W_{\varepsilon}}\left(\tau_{\varepsilon}\right)\right)\right)+\sum_{k=1}^{n}\left(D_{\tau, k} f\right)^{W}\left({ }^{\varepsilon} \mathcal{L} A_{k}\right)\left(\xi_{W_{\varepsilon}}(\lambda)\right)\right)\right]
\end{aligned}
$$

which as $\varepsilon \rightarrow 0$ gives rise to the equivalence

$$
\mathbb{E}\left[f \tilde{u}_{1}(t)\right] \simeq \frac{\varepsilon}{2} \mathbb{E}\left[\int_{t^{0}}^{t} \mathrm{~d} \tau\left(\sum_{r, k=1}^{n}\left(D_{\tau, r ; \tau, k}^{2} f\right)^{W}\left(\left(\mathbf{A}_{r} \cdot A_{k}\right)\left(\xi_{W}(\tau)\right)\right)+\sum_{k=1}^{n}\left(D_{\tau, k} f\right)^{W}\left({ }^{0} \mathcal{L} A_{k}\right)\left(\xi_{W}(\tau)\right)\right)\right] .
$$

Finally

$$
\mathbb{E}\left[f \tilde{u}_{2}(t)\right]=\mathbb{E}\left[\int_{t^{0}}^{t} f \cdot\left({ }^{W} \mathcal{R}_{0}-{ }^{W} Z\right) \mathrm{d} \tau\right]
$$

may be computed as before. There appear integrals along the paths of $D_{\tau, k} f, f$, and we get existence of $\hat{a}, \hat{b}, \hat{c}$ such that

$$
\lim _{\varepsilon \rightarrow 0} \frac{1}{\varepsilon} \mathbb{E}[\tilde{u}(t) f]=\int_{t^{0}}^{t} \mathbb{E}\left[\sum_{k, \ell}^{n} \hat{a}_{k \ell}\left(\xi_{W}(\tau)\right) D_{\tau, k ; \tau, \ell}^{2} f+\sum_{k=1}^{n} \hat{b}_{k}\left(\xi_{W}(\tau)\right) D_{\tau, k} f+\hat{c}\left(\xi_{W}(\tau)\right) f\right] \mathrm{d} \tau .
$$

It remains to take care of $\tilde{v}$ : combining (9) and (11) gives immediately $\tilde{v}=\mathrm{O}(\varepsilon)$. To get the sharper estimate $\mathrm{o}(\varepsilon)$ we use that (10) gives $\mathcal{R}_{k}^{1}$ as a bilinear expression in $u$. A typical term is

$$
\mathbb{E}\left[h \int_{t^{0}}^{t} \frac{W^{2} \partial^{2} A_{k}}{\partial \xi^{\ell} \partial \xi^{p}} u^{\ell} u^{p} \mathrm{~d} W^{k}\right]=\int_{t^{0}}^{t} \mathbb{E}\left[\mathcal{B}(\tau) u^{p}(\tau)\right] \mathrm{d} \tau, \quad \mathcal{B}:=\left(D_{\tau, k} h\right) \frac{{ }^{W} \partial^{2} A_{k}}{\partial \xi^{\ell} \partial \xi^{p}} u^{\ell} ;
$$


applying formula (14) and taking $f=\mathcal{B}$, we get

$$
\left|\mathbb{E}\left[h \int_{t^{0}}^{t} \frac{{ }^{0} \partial^{2} A_{k}}{\partial \xi^{\ell} \partial \xi^{p}} u^{\ell} u^{p} \mathrm{~d} W^{k}\right]\right| \leqslant c \varepsilon\|\mathcal{B}\|_{D_{2}^{s}} \leqslant c \varepsilon\|h\|_{\gamma_{3}^{2 s}}\|u\|_{\gamma_{2}^{2 s}},
$$

and using (9), with $\theta_{\varepsilon}(\cdot)$ replaced by $u$, we obtain the wanted order $\mathrm{O}(\varepsilon)$.

\section{References}

[1] V. Bally, D. Talay, The law of the Euler scheme for stochastic differential equations. I. Convergence rate of the distribution function, Probab. Theory Related Fields 104 (1996) 43-60.

[2] E. Gobet, Weak approximation of killed diffusion using Euler schemes, Stochastic Process. Appl. 87 (2000) 167-197.

[3] A. Kohatsu-Higa, S. Ogawa, Weak rate of convergence for an Euler scheme of nonlinear SDE's, Monte Carlo Methods Appl. 3 (1997) 327-345.

[4] D. Laurent, Analyse quasi-sure du schéma d'Euler, C. R. Acad. Sci. Paris 315 (1992) 599-602.

[5] D. Talay, L. Tubaro, Expansion of the global error for numerical schemes solving stochastic differential equations, Stochastic Anal. Appl. 8 (1990) 483-509.

[6] D. Talay, Z. Zheng, Quantiles of the Euler scheme for diffusion processes and financial applications, Math. Finance 13 (2003) 187-199.

[7] E. Teman, Analysis of error with Malliavin calculus: application to hedging, Math. Finance 13 (2003) 201-214.

[8] S. Watanabe, Analysis of Wiener functionals (Malliavin calculus) and its applications to heat kernels, Ann. Probab. 15 (1987) 1-39. 